\title{
Nonadiabatic analysis of strange-modes in hot massive stars with time-dependent convection
}

\author{
Takafumi Sonoi ${ }^{1,2, a}$ and Hiromoto Shibahashi ${ }^{2}$
}

1 Laboratoire d'études spatiales et d'instrumentation en astrophysique (LESIA), CNRS UMR8109, Université Pierre et Marie Curie, Université Denis Diderot, Observatoire de Paris, 5, Place Jules Janssen 92195 Meudon, France

2 University of Tokyo, School of Science, Department of Astronomy, Hongo 7-3-1, Bunkyo-ku, Tokyo, 113-0033, Japan

\begin{abstract}
We carry out nonadiabatic analysis of strange-modes in hot massive stars with time-dependent convection (TDC). We find that the instability of the modes excited at the Fe bump is weaker with TDC than with frozen-in convection (FC). But the instability still remains with TDC, and could be a possible candidate for the trigger of luminous blue variable (LBV) phenomena.
\end{abstract}

\section{Introduction}

"Strange-modes" were originally found by a theoritical study [1]. They are one type of stellar pulsation modes, but have significantly different properties from ordinary modes found in many observed pulsators. They appear only in very luminous stars with $L / M \gtrsim 10^{4} L_{\odot} / M_{\odot}$ [2], and have extremely short growth timescale, comparable with pulsation periods.

As for massive stars, [4] first suggested instability of strange-modes near the HumphreysDavidson (HD, [3]) limit. Because of the rapid growth of the amplitude, the instability might be associated to envelope expansion in luminous blue variables (LBV), and nonlinear analyses are going on to obtain the pulsationally-driven mass-loss (e.g. [5], [6], [7], [8]). A recent observation [9] found that the mass-loss rate in a luminous B star changes on a timescale of pulsation, which could be regarded as a strange-mode [10].

Stability of strange-modes has been so far analyzed with frozen-in convection (FC, e.g. [11], [12]). However, the Fe opacity bump in hot massive stars causes convection with certain contribution to energy transfer, and with timescale comparable to pulsation periods. Therefore, the convective effects on pulsation are not definitively negligible. In this study, we implement the time-dependent convection (TDC) formula by [13] to the nonadiabatic code by [14], and analyze the pulsational stability of radial modes in hot massive stars.

\section{TDC analysis}

Fig. 1 shows modal diagrams of radial modes in the $50 M_{\odot}$ model sequence with $X=0.70$, $Z=0.02$, constructed by MESA [15], in cases of FC and TDC. While the ascending sequences (e.g. A1, A2) correspond to ordinary modes, the descending ones to strange-modes. Instability on D1 and D2 sequences is weaker with TDC than with FC. For these sequences, the excitation takes place around the Fe bump convection zone. When taking into account TDC, damping effects due to the convection compensates the excitation, and weakens the instability.

a e-mail: takafumi.sonoi@obspm.fr

This is an Open Access article distributed under the terms of the Creative Commons Attribution License 4.0, which permits unrestricted use, distribution, and reproduction in any medium, provided the original work is properly cited. 

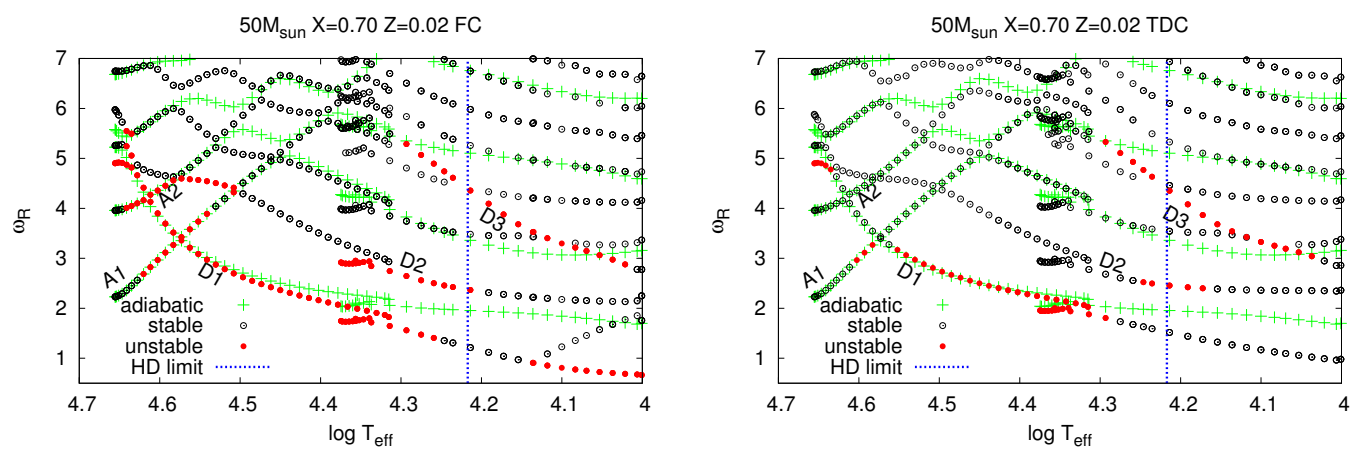

Fig. 1. Radial modes in the $50 M_{\odot}$ model sequence $(X=0.70, Z=0.02)$ for FC (left) and TDC (right). The crosses are modes obtained by the adiabatic approximation. The open and the filled circles are pulsationally stable and unstable modes, respectively, obtained by the nonadiabatic analysis. The vertical axis is nondimensional pulsation frequency multiplied by the dynamical timescale $\sqrt{R^{3} /(G M)}$. The horizontal axis is the logarithm of the effective temperature. The vertical dashed line indicates the evolutionary stage crossing the HD limit.

On the other hand, the instability of D3 is hardly different in cases of FC and TDC. For D3 sequence, the excitation occurs around the He opacity bump. The ratio of the convective to the total luminosity, $L_{C} / L_{r}$, is negligible, and the convective timescale is much longer than pulsation periods, while the ratio $L_{C} / L_{r}$ is non-negligible $\left(\sim 10^{-1}\right)$ and the convective timescale is comparable to pulsation periods in the Fe bump convection zone.

Fig. 1 also shows solutions by the adiabatic approximation. In the high effective temperature side, the D1 sequence fits to a sequence of adiabatic solutions. In this case, strange-modes are excited by the $\kappa$-mechanism. On the other hand, D1 sequence in the low effective temperature side, and D2 and D3 sequences do not have their corresponding adiabatic sequences. In this case, the so-called "strange-mode instability" acts on the modes (See [16] for details).

We have shown that TDC weakens the instability of the strange-modes. But the instability still remains with TDC, and could be a possible candidate for the trigger of LBV phenomena, although further nonlinear analyses are required.

\section{References}

1. Wood, P. R., Mon. Not. R. Astron. Soc., 174 (1976) 531

2. Shibahashi, H., Osaki, Y., Publ. Astron. Soc. Japan, 33 (1981) 427

3. Humphreys, R. M., Davidson, K., Astrophys. Journ., 232 (1979) 409

4. Kiriakidis, M., Fricke, K. J., Glatzel, W., Mon. Not. R. Astron. Soc., 264 (1993) 50

5. Dorfi, E. A., Gautschy, A., Astrophys. Journ., 545 (2000), 982

6. Chernigovski, S., Grott, M., Glatzel, W., Mon. Not. R. Astron. Soc., 348 (2004) 192

7. Grott, M., Chernigovski, S., Glatzel, W., Mon. Not. R. Astron. Soc., 360 (2005) 1532

8. Lovekin, C. C., Guzik, J. A. Mon. Not. R. Astron. Soc., 445 (2014) 1766

9. Aerts, C. et al., Astron. Astrophys., 513 (2010), L11

10. Godart, M. et al., Active $O B$ stars: structure, evolution, mass loss, and critical limits

(Proc. IAU Symposium No.272, San Franscisco: ASP, 2011) 503

11. Glatzel, W., Mehren, S. Mon. Not. R. Astron. Soc., 282 (1996) 1470

12. Saio, H., Georgy, C., Meynet, G. Mon. Not. R. Astron. Soc., 433 (2013), 1246

13. Grigahcéne, A. et al., Astron. Astrophys., 434 (2005), 1055

14. Sonoi, T., Shibahashi, H., Publ. Astron. Soc. Japan, 64 (2012), 2

15. Paxton, B. et al., Astrophys. Journ. Suppl., 208 (2013) 4

16. Sonoi, T., Shibahashi, H., Publ. Astron. Soc. Japan, 66 (2014), 69 